\title{
Caracterización del adulto mayor hospitalizado: un estudio retrospectivo
}

\author{
Edgar Camerino- Hernandez ${ }^{1, a}$; Tranquilina Gutierrez- Gómez ${ }^{2, b}$; \\ Maria Peñarrieta- De Cordova ${ }^{2, c}$; Maria Piñones- Martinez ${ }^{2, b}$.
}

\section{RESUMEN}

Objetivos: identificar las características del adulto mayor de 60 años, usuario de los servicios hospitalarios de internamiento del segundo nivel de atención durante los años 2006 al 2009. Material y métodos: el estudio fue documental, retrospectivo, realizado en el año 2011, se estudió al total de la población mayor de 60 años que recibieron atención en área de hospitalización de los Servicios de Medicina Interna, Cirugía, Ginecología y Traumatología entre los años 2006 al 2009, por medio del censo de la información capturada en el Sistema Automatizado de Egresos Hospitalarios (SAEH) del departamento de registros hospitalarios del Hospital Civil de Cd. Madero Tamaulipas. Resultados: el total de pacientes hospitalizados fue de 3422, el 60,4\% fueron mujeres, predominando el grupo de edad de 60-69 años con 1477 pacientes. Los servicios más utilizados fueron Medicina Interna y Cirugía, encontrándose que la Diabetes Mellitus y sus complicaciones representaron el porcentaje más alto $14,1 \%$ de las hospitalizaciones durante los 4 años, seguido de las cardiopatías con el 13,7\%. El 50,1\% de los pacientes permaneció de 1 a 3 días hospitalizados y el 82,8\% (2835) egresaron por mejoría. Conclusión: la información obtenida permite comprobar la efectividad de la política de salud actual en relación al otorgamiento de servicios de atención a la salud a los desprotegidos, que se refleja en una disminución de la utilización de los servicios de hospitalización del grupo etario en estudio.

PALABRAS CLAVE: Adulto mayor, pacientes hospitalizados, uso de servicios de salud.

\section{Characterization of elderly hospitalized: a retrospective study}

\section{SUMMARY}

Objectives: to identify the characteristics of senior citizens older than 60 who were users of inpatient hospital care at a secondary level hospital between 2006 and 2009. Material and Methods: It is a desk, prospective study conducted in 2011. The sample was the total population older than 60 years old who received care in an inpatient area of Internal Medicine, Surgery, Gynecology and Traumatology between 2006 and 2009 through a data census captured by the Hospital Discharge Automated System (SAEH) from hospital records department of the Civil Hospital of Madero Tamaulipas city. Results: the total number of hospitalized patients was 3422. A 60,4 \% were women; 1477 patients represented the group of 60-69 years old hence dominating the results. The most frequently used services were Internal Medicine and Surgery, finding that the Diabetes Mellitus and its complications represented $14.1 \%$, which is the highest percentage of hospitalizations in these 4 years, followed by heart disease with $13,7 \%$. A 50,1 $\%$ of patients remained hospitalized from one to 3 days and a $82,8 \%$ (2835) was discharged due to improvement. Conclusions: the gathered information allows checking the effectiveness of current health policy regarding the

Escuela de Enfermería, Universidad del Noreste. Tampico, Mexico.

Facultad de Enfermería de Tampico, Universidad Autónoma de Tamaulipas. Tampico, Mexico.

Licenciado en enfermeria. ${ }^{b}$ Doctora en enfermería. ${ }^{\mathrm{C}}$ Dra. en Ciencias de Salud Pública con mención en Sistemas de Salud. 
provision of services health care to the most vulnerable, reflected in a decrease in the use of inpatient services in the age group under study.

KEYWORDS: Senior citizens, Inpatient hospital services, use of health services

\section{INTRODUCCIÓN}

En nuestros días, el incremento de la población anciana y una mayor esperanza de vida, plantean grandes retos para el Sector Salud, debido a la alta prevalencia de enfermedades crónicas, su diagnóstico a edades más tempranas y a la producción de diversos tipos de incapacidades que en conjunto dan lugar a una demanda de servicios de salud mayor y compleja al involucrar diferentes niveles de atención. La utilización de los servicios de salud por parte de los diferentes grupos sociales, ha sido y es objeto de debate en muchos países, existen evidencias suficientes que demuestran que el proceso de utilización, se encuentra diferenciado socialmente y es explicado en buena parte por la existencia de desigualdades sociales en salud (1).

La Organización Mundial de la Salud, en el acuerdo de Kiev de 1979, con base a la esperanza de vida, consideró que las personas que viven en países desarrollados se les identifica como adultos mayores a partir de los 65 años de edad; la Asamblea Mundial del Envejecimiento, celebrada en Viena en 1982, en forma convencional consideró que los "ancianos" comprendían aquellas personas de 65 años de edad o más. En 1994 la Organización Panamericana de la Salud (OPS) ajustó la edad y señala como adulto mayor a la persona de 65 o más años de edad, con los datos anteriores, la clasificación según la información proporcionada, su finalidad y quien la exprese puede variar (2). En México, la Norma Oficial Mexicana NOM-167SSA1-1997 define a un adulto mayor como la persona de 60 años o más (3).

En 1930, en México la esperanza de vida al nacer en los hombres era de 35.5 años, al año 2000 aumentó a 73.1 años. De acuerdo a las estimaciones del censo de población 2010, el país registra que la población mexicana es de 112 322757 millones, de los cuales el 51,1\% son mujeres y el $48,8 \%$ son hombres, de tal manera que de cada 100 mexicanos 8 tienen 60 años o más. México ocupa el lugar número 11 en el mundo y el tercero de América por el monto de su población, superado por países como Estados Unidos de América y Brasil (4).

La diabetes mellitus, las enfermedades isquémicas del corazón, los tumores malignos, las enfermedades cerebro vasculares y las enfermedades crónicas de las vías respiratorias inferiores son las principales causas de muerte entre la población adulta mayor (4).

Actualmente según datos proporcionados por INEGI, la esperanza de vida en el año 2005, alcanzó los 75,4 años, las mujeres viven 77,9 años en promedio por 73 años de los varones. Entre 1970 y 2005 el indicador se incrementó en 14,5 años (5).

El incremento de la esperanza de vida es un indicador de mejoría del estado de salud de la población en edades tempranas, pero se relaciona en edades mayores con tasas elevadas de enfermedades crónicas y degenerativas e incapacidad que afectan su calidad de vida (6).

En Tamaulipas, la estructura por edad y sexo de la población se ha modificado de manera significativa, destacándose el inicio del proceso de envejecimiento demográfico que se expresa como un incremento relativo y absoluto de la población en edades avanzadas.

La Encuesta Nacional de la Dinámica Demográfica (ENADID), señala que Tamaulipas se encuentra entre los estados mexicanos que tienen el mayor porcentaje de adultos mayores en su estructura por edad, superado tan sólo por el Distrito Federal que tiene el 12 por ciento, Chihuahua con 11,8 por ciento, además de Yucatán, Veracruz y Nayarit que rondan el 11\%. A la vez la ENADID identifica que del total de hombres en Tamaulipas, $10,3 \%$ corresponde a personas de 60 años y más, y en el caso del total de mujeres $10,9 \%$ son de este grupo de edad; esto hace que la relación de hombres y mujeres sea de 86,9 hombres por cada 100 (7).

Para 2009, se estiman 122588 hombres y 144693 mujeres de 60 años y más; lo que significa una relación de hombres-mujeres de 85 hombres por cada 100 mujeres (5), de tal manera que de acuerdo con el Consejo Nacional de Población (CONAPO), para el 2011 el 8,4\% de los habitantes de Tamaulipas son adultos mayores, es decir, tienen 60 años y más de edad, entre 2005 y 2009, el número de adultos mayores pasó de 233176 a 267281 personas (8). Su tasa de crecimiento promedio anual en ese periodo fue de $3,47 \%$, mientras que la población total del estado 
creció a un ritmo de 1,27 por ciento, convirtiendo al estado de Tamaulipas, en uno de los estados con mayor porcentaje poblacional de adultos mayores.

Los servicios de salud que se prestan en los hospitales civiles de la Secretaría de Salud pueden considerarse la puerta de entrada al sistema de atención de la población civil que no tiene derecho a recibir atención a la salud en una institución pública del país. Para el adulto mayor que no tiene seguro de atención, esta institución pública puede ser la opción más accesible cuando presentan algún problema de salud o para recibir educación preventiva en salud.

Los estudios sobre uso de los servicios de salud en México son escasos, más aún aquellos que tratan de personas de 60 años o más, donde el problema se torna crítico debido al crecimiento de este grupo etario y las características propias del proceso de envejecimiento, cuando se demanda mayor atención de servicios de salud por la mayor frecuencia de padecimientos crónicos, su diagnóstico a edades más tempranas y la producción de distintos tipos de discapacidades, lo que dará lugar a una demanda de servicios de salud mayor y más compleja (9).

Aunque se conoce del incremento de la demanda de servicios de salud por la población mayor de 60 años en México, no existen suficientes datos respecto a la utilización específica de los servicios hospitalarios de segundo nivel de atención y de internamiento por dicho grupo poblacional, así como sus características distintivas, lo que da por consecuencia, la falta de información oportuna y detallada sobre los problemas de salud que requieren del uso de dichos servicios y que facilitaría intervenciones de prevención y diagnóstico temprano si se contara con ella, por lo anterior, el presente estudio pretende determinar las características distintivas de la población mayor de 60 años por servicio hospitalario de internamiento que utiliza, en una institución de salud de 2do.nivel de atención, durante los años 2006-2009.

La presente investigación se apoya en el modelo desarrollado por Anderson en 1995, que integra varias ideas acerca de cómo y por qué se usan los servicios de salud. El modelo sugiere un proceso causal considerando tres factores: 1) Factores Predisponentes, que a su vez comprenden aspectos demográficos (edad, sexo, estado civil, etc.) y aspectos de estructura social (apoyo familiar, tenencia de la vivienda y pensión de jubilación), 2) Factores capacitantes, que incluyen recursos comunitarios (aquellos que están disponibles para la población e influyen en el uso de los servicios como: ingreso, seguro de salud, fuente regular de cuidados y tiempo de espera), 3) Factores Organizacionales (satisfacción de la atención recibida, elección del médico tratante, tiempo de espera y proceso de citas)y 4) necesidad de salud: evaluar el propio estado de salud y decidir la búsqueda o no, de un especialista.

El modelo de comportamiento usa el concepto de mutabilidad para promover la equidad en el acceso. Para que una variable sea considerada como promotora del acceso debe ser mutable o modificable por el sistema de salud (10).

El objetivo del presente estudio de investigación fue identificar las características del adulto mayor de 60 años, usuario de los servicios hospitalarios de internamiento del segundo nivel de atención durante los años 2006 al 2009.

El adulto mayor de 60 años es una población vulnerable de escasos recursos económicos, en su mayoría sin seguro médico de atención, de donde se obtuvo información de factores predisponentes y capacitantes según Anderson. Se espera que los resultados sean de utilidad en el planeamiento de los servicios curativos y en la búsqueda de alternativas de atención de mejor calidad a menor costo para el adulto mayor.

\section{MATERIAL Y METODOS}

El estudio fue documental, retrospectivo, abarcó a toda la población mayor de 60 años que recibieron atención en área de hospitalización los servicios de Medicina Interna, Cirugía, Ginecología y Traumatología entre los años 2006 al 2009, por medio del censo de la información capturada en el Sistema Automatizado de Egresos Hospitalarios (SAEH) del departamento de registros hospitalarios del Hospital Civil de Cd. Madero Tamaulipas.

Se utilizó como instrumento el formato titulado $U>60 / 06-$ 09 donde se recolectó información obtenida de la base de datos referida y que abarca las siguientes variables operacionalizadas: Perfil de la población mayor de 60 años: integrado por las características demográficas que identifican a la población con 60 años o más, al momento del estudio y que incluyeron: edad, sexo, entidad, municipio, si cuentan con atención médica o servicio médico en Seguro Popular, Instituto Mexicano del Seguro Social (IMSS),Instituto de Seguridad y Servicio Social a los Trabajadores del Estado (ISSSTE), causas frecuentes de hospitalización con base al Sistema Automatizado de Egresos Hospitalarios (SAEH) del departamento de registros hospitalarios de la institución 
referida y acorde a la Secretaria de Salud: diagnóstico médico por el que se le indica tratamiento, incluye además, detección de infección intrahospitalaria, frecuencia de internamiento: número de veces en que se ha internado al año, promedio de días estancia por servicio: promedio de días de estancia hospitalaria por servicio desde el momento de su ingreso hasta el egreso, incluyendo servicio de ingreso, internamiento y permanencia causas de egreso: motivos por el cual fueron egresados o dados de alta.

Se realizó el análisis descriptivo utilizando el Excel y el inferencial con el programa estadístico SPSS versión 18.

\section{RESULTADOS}

El total de los pacientes hospitalizados de 60 años o más durante el periodo de 2006 a 2009 fue de 3422, el 60,4\% mujeres y $39,6 \%$ hombres.

El grupo de edad de mayor predominio fue de 60-69 años con 1477 pacientes de los cuales $614(41,57 \%)$ son hombres y 863 mujeres (58,42\%), el de 70-79 años de 1209 pacientes, el grupo de 80-89 con un total de 620 pacientes y el de 90 más con un total de 116 usuarios. Cabe mencionar que de entre el grupo de 90 a más, el rango máximo de la edad por año fue de 109 años (1 paciente) en el 2009, 104 años (1 paciente) en el 2007, 103 años (1 paciente) en el 2006 y 100 años (1 paciente) en el 2008, la mayoría mujeres.
Los servicios más utilizados por año, fueron Medicina interna y Cirugía, el resto de los servicios representaron un porcentaje muy bajo (menos del 1\%), de estos dos mismos servicios el más utilizado fue el de Medicina Interna con un total de 2166 usuarios de los cuales 795 fueron hombres y 1371 son mujeres.

El año con mayor número de hospitalizaciones fue el 2007 con 912 pacientes que representa el $26,7 \%$ del total de las hospitalizaciones en los cuatro años, predominando en el servicio de medicina interna por el sexo femenino. Sólo en el 2006 predomino el servicio de cirugía por hombres.

En relación a las principales causas de hospitalización durante el periodo de 2006 a 2009, se encontró que la Diabetes Mellitus y sus complicaciones representaron el porcentaje más alto con un $14,1 \%$ (483 pacientes) de las hospitalizaciones durante los 4 años ya mencionados, seguido de las cardiopatías con el $13,7 \%$ y como tercera causa el EPOC y otras enfermedades del sistema respiratorio, el resto de las causas de hospitalización se observa en la tabla 3. Para mostrar el tiempo que permanecieron hospitalizados los pacientes de 60 años o más, se establecieron tres rangos de días de hospitalización, de 1 a 3 días, de 4 a 6 días y de 7 o más días. El 50,1\% (1713 pacientes) de los pacientes permaneció de 1 a 3 días hospitalizado. El promedio de días de estancia hospitalaria más elevado por año fueron 74 días (1 paciente) en el 2009, 59 días (1 paciente) en el 2008, 55 días (1 paciente) en el 2006 y 34 días (1 paciente) en el 2007 (tabla 1).

Tabla 1. Causas más frecuentes de Hospitalización del año 2006 al 2009.

\begin{tabular}{|c|c|c|c|}
\hline Núm. & Causa & $f$ & $\%$ \\
\hline & Problemas relacionados con Diabetes Mellitus. & 483 & $14,1 \%$ \\
\hline & Cardiopatías. & 467 & $13,7 \%$ \\
\hline & EPOC y otras enfermedades del sistema respiratorio. & 270 & $7,8 \%$ \\
\hline & Diversos traumatismos por causas externas. & 223 & $6,5 \%$ \\
\hline & Enfermedades del sistema digestivo. & 194 & $5,7 \%$ \\
\hline & Tumores malignos y otros. & 178 & $5,2 \%$ \\
\hline & Colelitiasis y colecistitis. & 166 & $4,9 \%$ \\
\hline & I.R. y otras enfermedades del sistema urinario. & 128 & $3,8 \%$ \\
\hline & Hernias. & 127 & $3,7 \%$ \\
\hline & Enfermedades cerebro-vasculares. & 127 & $3,7 \%$ \\
\hline & Hiperplasia prostática. & 120 & $3,5 \%$ \\
\hline & Catarata senil. & 98 & $2,9 \%$ \\
\hline & Enf. del sistema osteo-muscular y tejido conjuntivo. & 86 & $2,5 \%$ \\
\hline & Enfermedades del hígado. & 83 & $2,4 \%$ \\
\hline & Hipertensión esencial o primaria. & 50 & $1,5 \%$ \\
\hline
\end{tabular}

Fuente. SAEH

$N=3422$ 


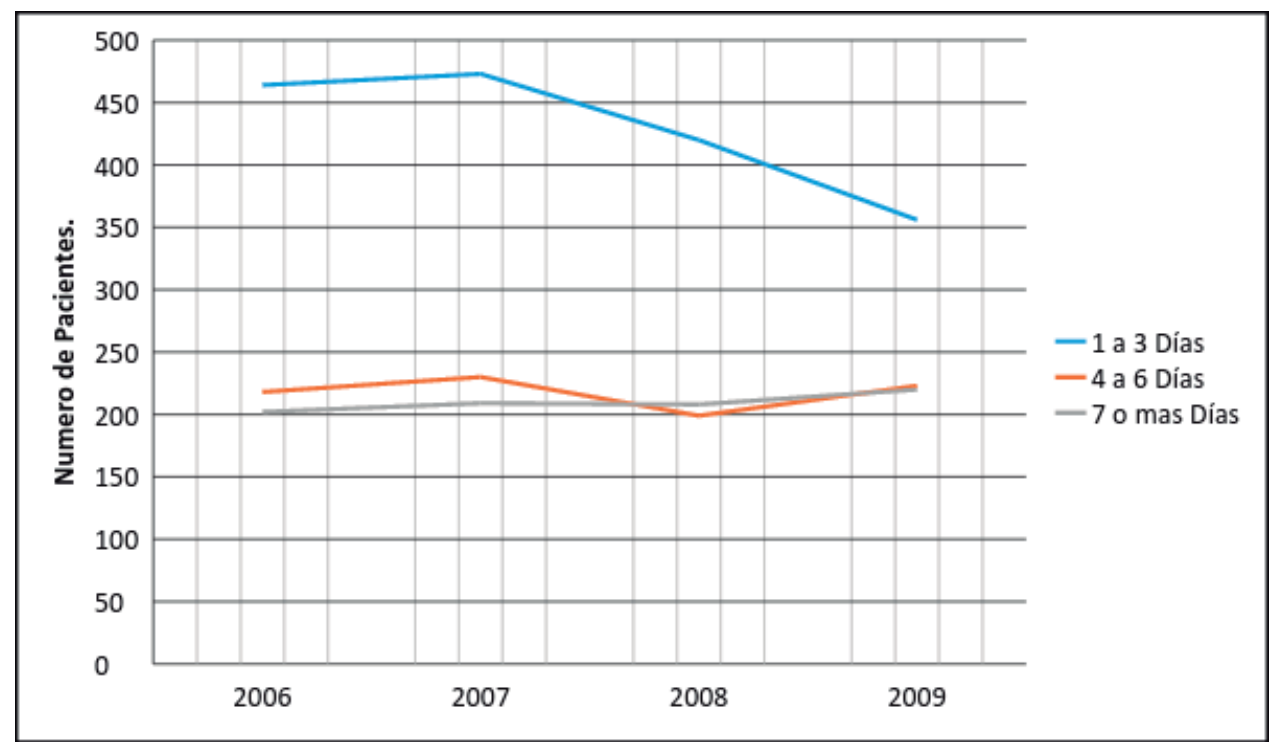

Fuente. SAEH

$N=3422$

Grafico 1. Días de hospitalización por años

En lo que se refiere a la atención a la salud, tenemos que 1777 pacientes lo cual representó el $54,2 \%$ de los pacientes, no tenían atención a la salud en ninguna institución, el 25,4\% (833 pacientes) contaban con Seguro Popular, 215 de los pacientes contaban con atención medica en el IMSS que representa el 6,5\%.

Referente a los motivos de egreso, en el $82,8 \%$ de los pacientes su egreso fue por mejoría, el 9,4\% de los pacientes egresaron por defunción.

Durante el periodo de 2006-2009 el número de mujeres de 60 años o más hospitalizadas fue mayor en comparación a los hombres. No se observó incremento de usuarios en los cuatro años de manera significativa, por lo contrario, se observó una disminución del 2007 al 2009. De igual forma, no se encontró diferencia estadística significativa entre los usuarios, según sexo y durante los cuatro años.

Al analizar días de estancia por años se encontraron diferencias estadísticas, en el rango de 1 a 3 días de hospitalización al comparar el 2006 con el 2009 hubo una disminución de los pacientes, lo cual puede interpretarse como un incremento en los días de hospitalización. La comparación de días de estancia por sexo permitió identificar que los hombres tuvieron más días de hospitalización con el 26,4\% (357 pacientes) en el rango de 7 o más días, que las mujeres con el $23,3 \%$ en el mismo rango (Grafico 1)

Por servicio de hospitalización, el 28,2\% (611 pacientes) de los pacientes hospitalizados en medicina interna requirieron de 7 o más días de estancia, mientras que el $62,9 \%$ de los pacientes de cirugía solo requirieron de 1 a 3 días.

Al cruzar el motivo de egreso con el sexo, se encontró que del total de egresos por mejoría que fue de 2835 las mujeres tienen el 61,4\% (1742 pacientes) y los hombres el $38,6 \%$ (1093 pacientes). El total de egresos por defunción es de 321 pacientes de los cuales las mujeres ocupan el índice mayor con un porcentaje del 53.3\% (171 pacientes) y los hombres tienen el 46.7\% (150 pacientes).

\section{DISCUSIÓN}

La geriatría se ha convertido en una necesidad de conocimiento y solo la experiencia del personal asistencial y de autoridades de la salud permitirá su necesario avance.

En el presente estudio realizado durante los años del 2006 al 2009, el total de adultos mayores hospitalizados fue de 3422 , de los cuales el $60,4 \%$ fueron mujeres, este dato es similar al encontrado en un estudio realizado en Chile, donde del total de adultos mayores hospitalizados (3486 adultos mayores), el 56\% fueron mujeres (11).

Al respecto, en una investigación realizada en la Unidad de Cuidados Intensivos (UCI) Polivalente del Hospital "Dr. Juan Bruno Zayas Alfonso" de Santiago de Cuba se encontró que de un total de 1707 pacientes ingresados entre los años 2002-2004, 794 tenían 60 años y más (46,5 $\%$ ), lo que demuestra como este indicador ha variado con 
los nuevos criterios de ingreso establecidos, donde la edad ya no constituye una limitante (12).

En relación a la utilización de servicios de salud según estudio realizado por Valdez et al., el $79 \%$ de los participantes reportaron contar con algún tipo de servicio médico para atender su salud,mencionando principalmente al Instituto Mexicano del Seguro Social (IMSS), seguido del Instituto de Seguridad Social al Servicio de los Trabajadores del Estado (ISSSTE) (13), así también Wong et al., encontraron que un poco más de la mitad de los adultos mayores dicen tener derecho a servicios médicos (14), en ambos casos difiere de lo encontrado en el presente estudio donde el $45,8 \%$ no recibe atención de salud en ninguna institución.

Chigne et al., en el estudio que realizaron en Perú en 1997, reportaron que durante los años de 1990 a 1996 el total de pacientes de 60 años o más que fueron hospitalizados fue de 3566 , dato muy similar al obtenido en el presente estudio donde el total de adultos mayores hospitalizados fue de 3422 durante el lapso de tiempo estudiado, también encontraron que las causas más frecuentes de hospitalización fueron las infecciones $(33,96 \%)$ y enfermedades del aparato circulatorio $(32,89 \%)$, dato que difiere en el presente estudio ya que se encontró que las causas más frecuentes de hospitalización son los problemas relacionados a la Diabetes Mellitus $(14,1 \%)$ y cardiopatías $(13,7 \%)(15)$.

El grupo de edades de mayor predominio en el presente estudio fue de 60-79 con el 78,4\%, dato similar al encontrado en el estudio realizado en Pinar del Río, Cuba en el 2003, donde el grupo etario más relevante fue el de 60 a 74 años de edad con un $64 \%$ (16); que a su vez también es similar al encontrado en el estudio realizado Costa Rica en el 2007 donde el grupo de edad de mayor predomino fue el de 65 a 69 años conformado por una tercera parte del total de la población de adultos mayores (17).

En relación a los egresos hospitalarios, se observó que, de 2835 egresos por mejoría, fueron mujeres $1742(61,4 \%)$ y $1093(38,6 \%)$ hombres, dato que difiere del encontrado por Martínez y Garmendia (18) en Chile, cuyo estudio no refiere diferencias en sexo en el número de egresos hospitalarios.

\section{CONCLUSIONES}

En relación al número de pacientes hospitalizados de 60 años o más durante el periodo 2006 a 2009 fue de predominó del sexo femenino.

El rango máximo de edad fue de 109 años. Los adultos mayores de 100 años en su mayoría fueron mujeres.

El grupo de edad de mayor predominio fue de 60-69 años siendo en su mayoría mujeres. El año en que se registraron mayor número de hospitalizaciones para este grupo etario fue el 2007, predominando en el servicio de medicina interna, a su vez este servicio fue donde los usuarios requirieron más días de estancia hospitalaria.

La principal causa de hospitalización del 2006 al 2009 fue la Diabetes Mellitus y sus complicaciones, demostrando que se debe tener mayor vigilancia o mejor control sobre los usuarios que padecen esta enfermedad crónica.

En cuanto a los días que permanecen hospitalizados los usuarios de 60 años o más, la gran mayoría solo requirió de 1 a 3 días de estancia hospitalaria, pero también se encontró que hubo un incremento en los días de hospitalización siendo los hombres los que requirieron de más días de estancia.

En su mayoría los pacientes que son atendidos en la institución estudiada no cuentan con un servicio médico, al comparar el 2006 con el 2009 se detectó un incremento de usuarios que cuentan con seguro popular.

La información obtenida en el presente estudio permitió comprobar la efectividad de la política de salud actual; en relación al otorgamiento de servicios de atención a la salud a los desprotegidos, que se refleja en una disminución de la utilización de los servicios de hospitalización del grupo etario en estudio.

\section{REFERENCIAS BIBLIOGRAFICAS}

1. Molina R, Poblano O, Carrillo, C, Sauceda A. Utilización de servicios de salud en México. Salud en Tabasco. 2006; 12(1): 427-432.

2. Novelo de López H. Aspectos demográficos del adulto mayor. Revista de nutrición clínica. 2003; 6(1): 63-69.

3. Estados Unidos Mexicanos, Secretaría de Salud. Norma oficial mexicana NOM-167-SSA1-1997. Norma Oficial Mexicana, para la prestación de servicios de asistencia social para menores y adultos mayores. Ciudad de Mexico: Secretaría de Salud; 1997. (Citado en junio del 2016) Disponible en: http://www.salud.gob.mx/unidades/cdi/ nom/167ssa17.html

4. Instituto Nacional de Estadística y Geografía. Mujeres y Hombres en México. Décima tercera edición. Ciudad de Mexico: INEGI; 2009. 
5. Instituto Nacional de Estadística y Geografía. Esperanza de vida por entidad federativa según sexo. Ciudad de Mexico: INEGI; 2010.

6. Shamah-Levy T, Villalpando-Hernández S, RiveraDommarco JA. Resultados de nutrición de la ENSANUT 2006. Cuernavaca, México: Instituto Nacional de Salud Pública; 2007.

7. Zertuche R. Crece 10,7\% el número de ancianos en Tamaulipas en los últimos 20 años. Tamaulipas: Gaceta; 2010.

8. Consejo Nacional de Población. Tamaulipas: indicadores demográficos 1990-2030. Ciudad de Mexico: CONAPO; 2002.

9. Peñarrieta MI, Mercado EJ, Piñones S, Gutiérrez T, del Ángel B. Factores que intervienen en la utilización de los servicios de salud por el adulto mayor Tampico - Tamaulipas - México 2003-2004. Investigación y Educación en Enfermería. 2006; 24(2):30-38.

10. Andersen R. Revisiting the behavioral model and access to medical care. Does it matter. J Health Soc. 1995;36(1): 1-10.

11. Marín P, Chávez P, Carrasco M, Gac H, Alonso C, Rodríguez L. Utilización del servicio de urgencia de un hospital universitario por los adultos mayores en Santiago de Chile. Revista Española de Geriatría y Gerontología. 2009; 46(1):27-29.

12. Rodríguez O. Morbilidad y Mortalidad en pacientes ingresados en Unidad de Cuidados Intensivos (2002-2004).

Tesis para optar por el título de Máster en Urgencias Médicas. 2007. Santiago de Cuba: Hospital General Docente Dr. Juan Bruno Zayas Alfonso; 2007.

13. Valdez EA, Román R, Cubillas MJ. Análisis de la oferta de servicios para adultos mayores y sus necesidades manifiestas. Psicología y Salud. 2005; 15(1): 127-133.

14. Wong R, Espinoza M, Palloni A. Adultos mayores mexicanos en contexto socioeconómico amplio: salud y envejecimiento. Salud Pública de México. 2007; 49(4):S436-S447.
15. Chigne O, Varela L, Chávez H, Sandoval L. Utilización de los servicios de hospitalización, consulta externa y emergencia, por adultos mayores y de menor edad, en el Hospital Nacional Cayetano Heredia. Rev Med Hered. 1997; 10: 111-118.

16. Figueredo N, Sotolongo I, Arcias R, Díaz G. Caracterización del adulto mayor en la comunidad. Rev Cubana Enfermería. 2003; 19 (3):0-0.

17. Chaves O, León M. Accesibilidad y uso de los servicios de salud en la población adulta mayor de Costa Rica. San Jose de Costa Rica: Dirección de Compra de Servicios de Salud/Gerencia de División Administrativa/Caja Costarricense de Seguro Social; 2007.

18. Martínez MS, Garmendia ML. Inequidades en la utilización de servicios de hospitalización en personas mayores en Chile. Santiago: Expansiva; 2008.

\section{Correspondencia:}

Tranquilina Gutiérrez Gómez

Correo electrónico: tgutierr@uat.edu.mx

Fecha de Recepción: 18 de abril del 2016. Fecha de aceptación: 29 de junio del 2016. 